\title{
An Elementary Study of a Class of Dynamic Systems with Single Time Delay
}

\author{
Akio Matsumoto \\ Chuo University, \\ Department of Economics, \\ 742-1, Higashi-Nakano, Hachioji, Tokyo, 192-0393, Japan. \\ akiom@tamacc. chuo-u. ac.jp \\ and \\ FERENC SZIDAROVSZKY \\ University of Pecs, \\ Department of Applied Mathematics, \\ Pecs, Ifjusag u. 6, H-7624, Pecs,Hungary \\ szidarka@gmail.com
}

\begin{abstract}
A complete eigenvalue analysis is given for a certain class of dynamic systems with a single delay. The stability region is determined and it is demonstrated that there is only one stability switch. Special cases from economics, biology and engineering illustrate the importance of such models.
\end{abstract}

\section{RESUMEN}

Un análisis completo de los autovalores se entrega para una clase de sistemas dinámicos con retardo simple. La región de estabilidad se determina y se demuestra que existe solamente un switch de estabilidad. Casos especiales para Economía, Biología e Ingeniería ilustran la importancia de los modelos mencionados.

Keywords and Phrases: dynamic systems, time delay, stability.

2010 AMS Mathematics Subject Classification: 34K20, 37C75. 


\section{Introduction}

In examining economic and engineering systems we often face with delayed data and delayed responses. In the case of fixed delays the system is described by a difference-differential equation and in the case of continuously distributed delay the model is a Volterra-type integro-differential equation. Delay models have many applications in engineering, biology and economics to name only the most important fields (Hale (1979); Cushing (1977); Invernizzi and Medio (1991)).

Without time delay the governing dynamic model is a system of ordinary differential equations, the asymptotical behavior of the solution trajectories can be examined by well established methods such as the usage of Lyapunov functions and local linearization. If the system is linear, then local asymptotical stability implies global stability, and the spectrum is finite making the analytic investigation relatively simple. In the case of continuously distributed delay with gamma-density weighting functions, the spectrum remains finite, however for fixed delays the spectrum is usually infinite. In the case of linear systems with fixed delays the characteristic equation is an exponentialpolynomial equation. There is a large literature on delayed equations with one delay (see for example, Hayes (1950) and Burger (1956) for the earliest studies), however only very few studies are devoted to multiple delays (see for example, Hale and Huang (1993) and Piotrowska (2007)).

In this paper we will consider a special case of nonlinear dynamics with one delay and will present an elementary analysis of its spectrum which can lead to a complete understanding of its local asymptotical behavior.

\section{Practical Examples}

Consider first a monopoly where one firm produces a product and sells it to a homogeneous market. Let $x$ be the production output of the firm and $p(x)=a-b x(a, b>0)$ the price function. If the firm determines its production level based on a delayed price information, then it is $a-b x(t-\tau)$, where $\tau$ is the delay. Assuming gradient adjustment process in the dynamics, in the absence of time delay the governing dynamic equation would be as follows:

$$
\dot{x}(t)=\alpha(x(t))(a-c-2 b x(t)),
$$

since the profit is given as

$$
\varphi=x(a-b x)-c x
$$

where $c$ is the firm's marginal cost. In the presence of delay, equation (1) has to be modified as

$$
\dot{x}(t)=\alpha x(t)(a-c-2 b x(t-\tau))
$$

where we assume that $\alpha(x)=\alpha x$ with a positive coefficient $\alpha$. The only positive steady state of the system is

$$
\bar{x}=\frac{a-c}{2 b} .
$$


In order to guarantee that this output level is positive, we have to assume that $a>c$. Linearizing equation (3) around $\bar{x}$ and introducing the new variable $z=x-\bar{x}$ a single delay ODE is obtained:

$$
\dot{z}(\mathrm{t})=-\gamma z(\mathrm{t}-\tau)
$$

where $\gamma=\alpha(a-c)>0$.

Consider next an electrical system with state feedback, where the feedback is delayed. Assume the systems equation is linear:

$$
\dot{x}(t)=A x(t)+B u(t)
$$

where $\boldsymbol{x}$ is the state and $\mathbf{u}$ is the input. Let $\mathbf{K}$ be the feedback matrix and $\tau$ the delay. Then the delayed feedback system can be written as

$$
\dot{x}(t)=A x(t)+B(u(t)+K x(t-\tau)) .
$$

In the single-dimensonal case this equation reduces to the following:

$$
\dot{x}(t)=A x(t)+B K x(t-\tau)+B u(t)
$$

In the special case of constant input, $u(t) \equiv u_{0}$, and $A=0$ introduce the new variable $z=x+u_{0} / K$ to have

$$
\dot{z}(\mathrm{t})=\mathrm{BK} z(\mathrm{t}-\tau),
$$

which has the same form as equation (4) with $\gamma=-\mathrm{BK}$.

Models in population dynamics are often delayed equations, when reproduction is not instantenuous. Assuming exponential growth rate, the model can be written as

$$
\dot{x}(t)=r x(t-\tau)
$$

where $r$ is the reproduction rate and $\tau$ is the delay. Notice that this equation also has the form as (4) with $r=-\gamma$.

\section{Spectrum Analysis}

As usual, we look for the solution in the exponential form $z(t)=e^{\lambda t} v$, and substitute it into equation (4) to get

$$
\lambda+\gamma e^{-\lambda \tau}=0
$$

Multiplying by $\tau$ and introducing the new variables $\Delta=\lambda \tau$ and $A=\gamma \tau$, this equation is simplified as

$$
\Delta+A e^{-\Delta}=0 .
$$

Assume that $\Delta=\alpha+i \beta$ is a complex root. Then

$$
\alpha+i \beta+A e^{-\alpha}(\cos \beta-i \sin \beta)=0 .
$$


Equating the real and imaginary parts with zero,

$$
\alpha+A e^{-\alpha} \cos \beta=0
$$

and

$$
\beta-A e^{-\alpha} \sin \beta=0
$$

From (11),

$$
e^{-\alpha}=\frac{\beta}{A \sin \beta},
$$

if $\sin \beta \neq 0$. If $\sin \beta=0$, then from (9), $\Delta$ is real and therefore is the solution of the real equation

$$
\mathrm{Ae}^{-\Delta}=-\Delta
$$

Depending on the value of $A$, there is either no solution, or 1 or 2 negative solutions. So the real solutions (if exist) are negative. Assume now that $\sin \beta \neq 0$, then from (10) and (12),

$$
\alpha+A \frac{\beta}{A \sin \beta} \cos \beta=0
$$

showing that

$$
\alpha=-\beta \cot \beta \text {. }
$$

Without losing generality we may assume that $\beta>0$, since if $\Delta$ is a solution of equation (9), then its complex conjugate is also a solution. From (13) we see that the real part of $\Delta$ is negative if and only if

$$
\beta \in\left(n \pi, \frac{\pi}{2}+n \pi\right), n=0,1,2, \ldots
$$

Substituting (13) into relation (11), we get a single-variable equation for $\beta$ :

$$
\frac{1}{A} \beta=e^{\beta \cot \beta} \sin \beta \text {. }
$$

Let $f(\beta)$ denote the right hand side of this equation. We will next examine the shape of the graph of this function. Clearly

$$
\lim _{\beta \rightarrow 0} f(\beta)=0
$$

and for $n \geq 1$,

$$
\lim _{\beta \rightarrow n \pi-0} f(\beta)=0,
$$

since $\beta \cot \beta$ converges to $-\infty$ as $\beta$ tends to $n \pi$ from the left. Similarly

$$
\lim _{\beta \rightarrow n \pi+0} f(\beta)=\left\{\begin{array}{cc}
\infty & \text { if } n \text { is even } \\
-\infty & \text { if } n \text { is odd }
\end{array}\right.
$$

since the value of $K=\beta \cot \beta$ tends to $\infty$ as $\beta$ tends to $n \pi$ from the right and

$$
f(\beta)=e^{k} \frac{1}{K} \beta \cos \beta \text {. }
$$


In addition,

$$
\lim _{\beta \rightarrow \frac{\pi}{2}+n \pi} f(\beta)=\left\{\begin{array}{cc}
1 & \text { if } n \text { is even } \\
-1 & \text { if } n \text { is odd }
\end{array}\right.
$$

since $K \rightarrow 0$ here. Simple differentiation shows that

$$
\begin{aligned}
f^{\prime}(\beta) & =\cos \beta e^{\beta \frac{\cos \beta}{\sin \beta}}+\sin \beta e^{\beta \frac{\cos \beta}{\sin \beta}} \frac{(\cos \beta-\beta \sin \beta) \sin \beta-\beta \cos ^{2} \beta}{\sin ^{2} \beta} \\
& =\frac{1}{\sin \beta} e^{\beta \frac{\cos \beta}{\sin \beta}}[\sin 2 \beta-\beta]
\end{aligned}
$$

Notice that there is a unique $\beta^{*} \in(0, \pi / 2)$ such that $\sin 2 \beta^{*}=\beta^{*}$, and for $\beta<\beta^{*}, \sin 2 \beta>\beta$ and for $\beta>\beta^{*}, \sin 2 \beta<\beta$. Therefore

$$
f^{\prime}(\beta)>0 \text { if and only if either } \beta \in\left(0, \beta^{*}\right) \text { or } \beta \in((2 k-1) \pi, 2 k \pi), k=1,2, \ldots
$$

and

$$
f^{\prime}(\beta)<0 \text { if and only if either } \beta \in\left(\beta^{*}, \pi\right) \text { or } \beta \in(2 k \pi,(2 k+1) \pi), k=1,2, \ldots
$$

The graph of $f(\beta)$ is shown in Figure 1. The value of $\beta$ is the intersection of this graph with the linear function $\beta / A$. Assume first that $A>\pi / 2$. Then function $\beta / A$ crosses the $\beta=\pi / 2$ vertical line under one, so there is a root between $\pi / 2$ and $\pi$. Here the value of $\alpha$ is positive making the system unstable regardless of the other solutions. If $A<\pi / 2$, then there is no intersection between $\pi / 2$ and $\pi$, however depending on the value of $A$ there is the possibility of solution between 0 and $\pi / 2$, where $\alpha$ is negative.

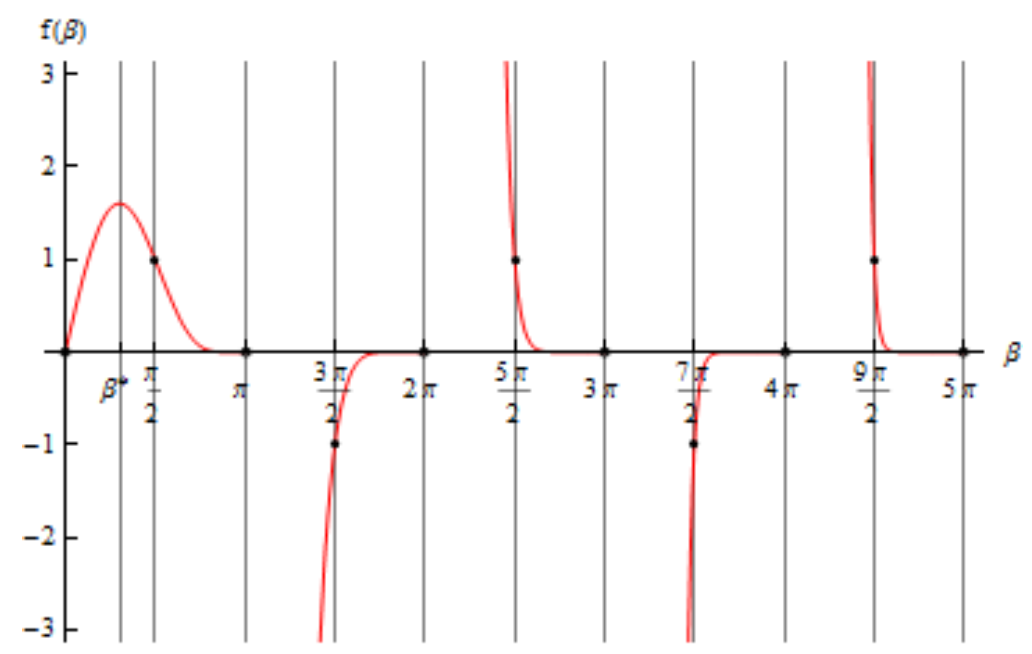

Figure 1. Shape of the graph of $f(\beta)$ 
Notice that in this case line $\beta / A$ crosses the $\beta=\pi / 2$ vertical line above one, so the other intersections with the graph of $f(\beta)$ are in intervals $\left(2 k \pi, \frac{\pi}{2}+2 k \pi\right), k=1,2, \ldots$, where the corresponding $\alpha$ value is negative. Hence we have the following result.

Proposition 3.1. Assume $A>0$. Then the system (9) is asymptotically stable if $A<\frac{\pi}{2}$, and unstable if $A>\frac{\pi}{2}$.

Consider next the case of $A<0$ in equation (9). If $\sin \beta=0$, then equation (11) implies that $\beta=0$, so $\Delta$ is real, and solves equation

$$
\Delta=-\mathrm{Ae}^{-\Delta}
$$

This equation always has a positive solution, so the system is always unstable.

\section{$4 \quad$ Stability Switches}

Assume again that $A>0$. Stability switches are usually examined by looking for pure complex eigenvalues $\Delta=i \beta$ ( $\beta>0$ since complex conjugate is also a solution). With $\alpha=0$, equations (10) and (11) are reduced to the more simple equations

$$
A \cos \beta=0
$$

and

$$
\beta-A \sin \beta=0
$$

From (17),

$$
\beta=\frac{\pi}{2}+n \pi
$$

however from (18), $\sin \beta$ has to be positive, so

$$
\beta=\frac{\pi}{2}+2 n \pi(n=0,1,2, \ldots)
$$

are the stability switches with the corresponding values of $A=\beta$. In order to detect the direction of the stability switches we consider $\Delta$ as the function of the bifurcation parameter $A$. Implicitly differentiating equation (9) with respect to $A$ gives

$$
\Delta^{\prime}+e^{-\Delta}-A e^{-\Delta} \Delta^{\prime}=0
$$

showing that

$$
\Delta^{\prime}=\frac{e^{-\Delta}}{A e^{-\Delta}-1}=\frac{\Delta}{A \Delta+A}=\frac{i \beta}{i \beta A+A}=\frac{i \beta(A-i \beta A)}{A^{2} \beta^{2}+A^{2}}
$$

with real part $\beta^{2} /\left(A \beta^{2}+A\right)>0$ showing that the real part of the eigenvalue changes from negative to positive. Notice that $A=\pi / 2$ is the only stability switch, since for all $A=\frac{\pi}{2}+2 n \pi(n \geq 1)$ there is an eigenvalue with positive real part with $\beta \in(\pi / 2, \pi)$ so regardless what happens with the other eigenvalues the system is unstable anyway. At $A=\frac{\pi}{2}$ Hopf bifurcation occurs giving the possibility of the birth of limit cycles. 


\section{Conclusions}

A special class of dynamic systems was examined where a single delay was present. Based on elementary analysis the spectrum of the system was completely described and the stability region characterized. We proved that the system is asymptotically stable if $A<\frac{\pi}{2}$ and unstable if $A>\frac{\pi}{2}$. If $A=\frac{\pi}{2}$, then Hopf bifurcation occurs. In the model of a monopolistic firm $A=\lambda \tau$, where $\tau$ is the delay and $\gamma$ is the product of the marginal speed of adjustment and the difference of the maximum price and marginal cost. Since both $\gamma$ and $\tau$ are positive, the stability region is the domain between the positive branch of the hyperbola $\tau=\frac{\pi}{2 \gamma}$ and the $\gamma=0$ positive horizontal axis. Similar interpretation can be given for the delay electrical systems and for the delay population dynamic models in the paper.

We also demonstrated that there are infinitely many values of $A$ which correspond to pure complex eigenvalues, however the smallest such value is the only stability switch. The case of multiple delays is much more complicated. It will be the subject of our future study.

Received: July 2011. Accepted: October 2012.

\section{References}

[1] Burger, E. (1956), On the Stability of Certain Economic Systems. Econometrica, 24(4), 488-493.

[2] Cushing, J. M. (1977), Integro-Differential Equations and Delay Models in Population Dynamics. Springer-Verlag, Berlin/Heidelberg/New York.

[3] Hale, J. (1979), Nonlinear Oscillations in Equations with Delays. In Nonlinear Oscillations in Biology (K. C. Hoppenstadt, ed.), Lectures in Applied Mathematics, 17, 157-185.

[4] Hale, J. and W. Huang (1993), Global Geometry of the Stable Regions for Two Delay Differential Equations. J. of Math. Analysis and Appl., 178, 344-362.

[5] Hayes, N. D. (1950), Roots of the Transcendental Equation Associated with a Certain Difference-Differential Equation. J. of the London Math. Society, 25, 226-232.

[6] Invernizzi, S. and A. Medio (1991), On Lags and Chaos in Economic Dynamic Models. Journal of Math. Econ., 20, 521-550.

[7] Piotrowska. M. (2007), A Remark on the ODE with Two Discrete Delays. Journal of Math. Analysis and Appl., 329, 664-676. 